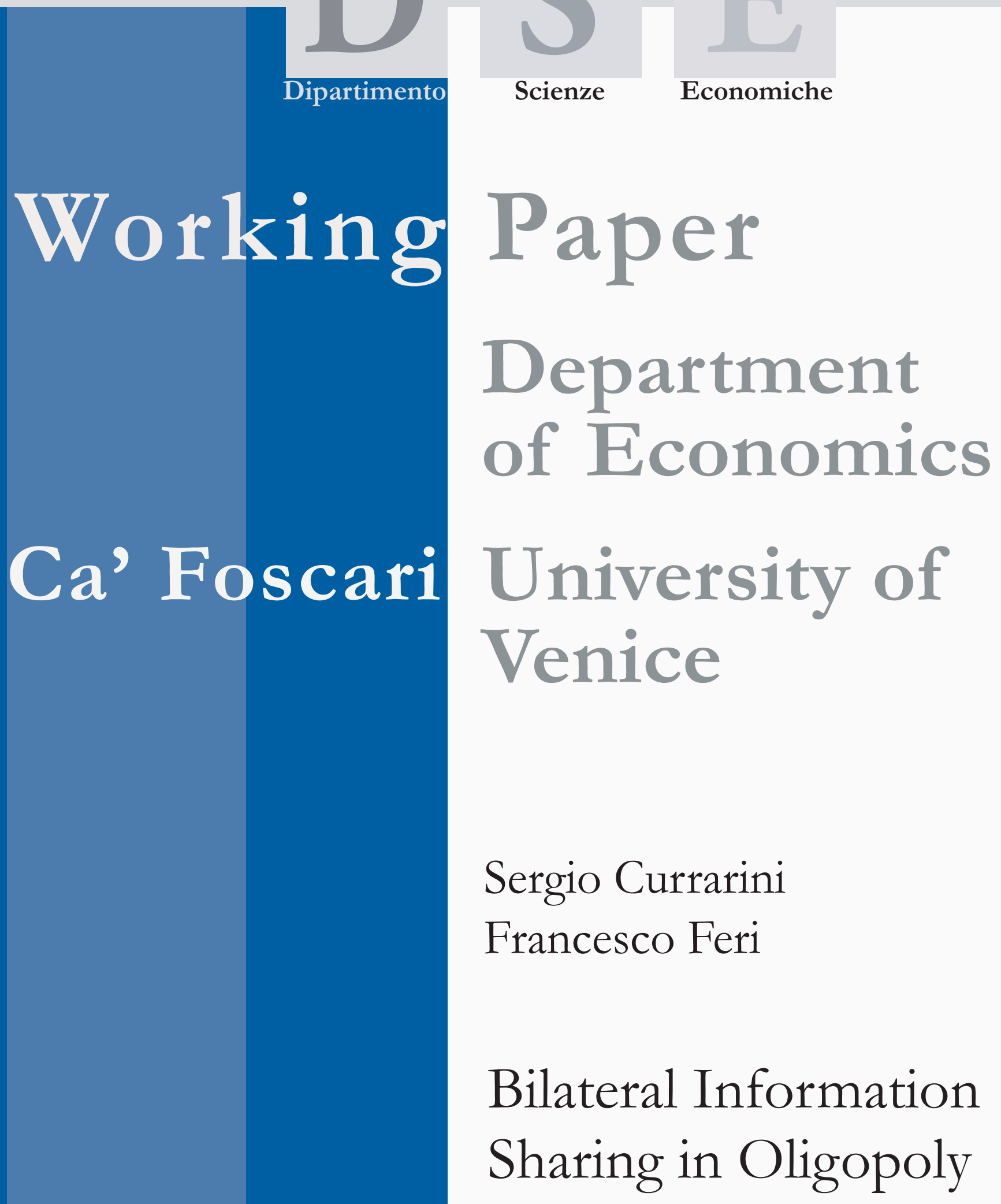




\title{
Bilateral Information Sharing in Oligopoly
}

\author{
Sergio Currarini \\ Università Ca' Foscari di Venezia \\ Francesco Feri \\ University of Innsbruck
}

October 2007

\begin{abstract}
We study the problem of information sharing in oligopoly, when sharing decisions are taken before the realization of private signals. Using the general model developed by Raith (1996), we show that if firms are allowed to make bilateral exclusive sharing agreements, then some degree of information sharing is consistent with equilibrium, and is a constant feature of equilibrium when the number of firms is not too small. Our result is to be contrasted with the traditional conclusion that no information is shared in common values situations with strategic substitutes - such as Cournot competition with demand shocks - when firms can only make industry-wide sharing contracts (e.g., a trade association).
\end{abstract}

Keywords Information sharing, oligopoly, networks, Bayesian equilibrium.

JEL Codes D43, D82, D85, L13

Address for correspondence:

Sergio Currarini

Department of Economics

Ca’ Foscari University of Venice

Cannaregio 873, Fondamenta S.Giobbe

30121 Venezia - Italy

Phone: (++39) 0412349133

Fax: (++39) 0412349176

e-mail: email

This Working Paper is published under the auspices of the Department of Economics of the Ca' Foscari University of Venice. Opinions expressed herein are those of the authors and not those of the Department. The Working Paper series is designed to divulge preliminary or incomplete work, circulated to favour discussion and comments. Citation of this paper should consider its provisional character.

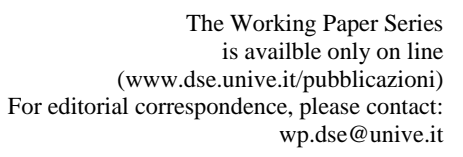




\section{Introduction}

The incentives of oligopolistic firms to share private information when facing an uncertain environment have been the object of a large literature, pioneered by the works of Novshek and Sonnenschein (1982), Clarke (1983), Vives (1985) and Gal-or (1985)). Such incentives have turned out to vary across the various models of oligopolistic competition, and to delicately depend on specific assumptions, making the construction of a general theory problematic.

The general model proposed by Raith (1996) has shown, however, that such differences can be basically imputed to the nature of the private information held by firms (which can be of the common value or private value type and can have various degrees of precision) and to the type of strategic interaction on the market (where strategies can be complements or substitutes).

In terms of economic policy, understanding the incentives to share information is of crucial importance. When no such incentives exist, any evidence of information sharing should be interpreted as evidence of collusion, with obvious implication for social welfare. This has in fact been the line followed by Kühn and Vives (1995) in their study of the EU industry.

As shown in several works on this topic and effectively summarized by Raith (1996), the absence of information is a typical feature of common value problems in which strategies are substitutes (therefore including the standard Cournot model). This result has been obtained in various forms and in two different models of information transmission.

The "strategic" model studies a simultaneous game of information revelation, in which firms decide whether to disclose or to withhold information. In this model, the decision to withhold one's own information does not affect the acquisition of the private information disclosed by other firms. Withholding information is the unique dominant strategy of this game in all common value situations with strategic substitutes. In contrast, when private information is not correlated across firms (private value) or, even if correlated, contains no noise (perfect signals), revealing information is always a dominant strategy (see Raith, 1996).

In the "contractual" model, sharing contracts are "exclusive", by this meaning that firms exchange information on a quid pro quo basis (see Clarke, 1983 and Kirby, 1988). Here, all firms that do not reveal their private information are excluded from the information revealed by other firms. This model has lent itself to the analysis of a simple mechanisms (e.g., an industry-wide trade association) which universally discloses information if and only if all firms have chosen to disclose, otherwise no information is shared. Information sharing takes place 
in equilibrium when the expected profits of firms are higher than when using only their own private signal. As shown in Raith (1996), under common value and strategic substitutes firms prefer the complete absence of information to an industry-wide sharing contract, as long as products differentiation is not too high. ${ }^{1}$

In this paper we will study a different contractual model, in which firms share information on the basis of exclusive bilateral contracts. Information sharing is therefore no longer mediated by a centralized industry-wide agency, but goes through private arrangements of entrepreneurs. The restriction to bilateral contracts does not, in principle, rule out equilibria in which information sharing groups arise, and allows for the emergence of other, more complex, information structures, that can be usefully thought of as a network of sharing agreements.

We define a pairwise stable information structure as a set of sharing agreements with the property that no new agreement is formed and no existing one is severed. ${ }^{2}$ Our main result is contained in proposition 1 and refers to common value situations. We show that the absence of information sharing (the empty network in the present context) is never a pairwise stable structure, even when strategies are substitutes and products are homogeneous (the standard Cournot game). For those situations in which complete information pooling (the complete network) is Pareto dominated by the absence of information sharing, this result is obtained under the additional condition that the number of firms in the market is not too small.

The intuition underlying this result is quite simple. Consider the decision of two firms, say $i$ and $j$, to share their private information when no other firm is in the market. This decision has two distinct effects on the expected profits of, say, firm $i$. The first (positive) effect stems from the refinement of $i$ 's expectation the state of the world and from the observation of $j$ 's signal; the second (negative) effect stems from the revelation of $i$ 's signal to firm $j$. As discussed above, the net effect is negative as long as products are not too differentiated (see Raith, 1996).

Suppose now that other firms are in the market. Now, the information shared by $i$ and $j$

\footnotetext{
${ }^{1}$ For the standard Cournot game with homogeneous goods, Kirby (1988) has also looked at a different contract, in which firms can decide to infringe the contract, expecting all other firms to keep sharing their own information. He finds that the industry-wide agreement is stable whenever it Pareto dominates the absence of information sharing. The same type of contract was considered in Vives (1990) for the case of monopolistic competition.

${ }^{2}$ The concept of pairwise stability, adapted here to information structures, has been defined for general network problems by Jackson and Wolinsky (1996).
} 
has the additional effect of refining both $i$ 's and $j$ 's expectation on the private information held by the other firms (and, thereby, on their equilibrium behavior). This refinement is due to the correlation of firms signals, and has a positive effect on both $i$ 's and $j$ 's expected profits. Note also that this additional information is acquired by $i$ and $j$ without revealing to the other firms any information about $i$ 's and $j$ 's signals (in this sense, at no cost).

The magnitude of this second effect clearly increases with the number of firms in the market, from which the requirement of proposition 1 on the number of competitors. It must be pointed out, however, that the presence of information sharing in equilibrium can be compatible with the minimal number of firms (three) for which the additional effect underlying our proposition is generated. This is shown by means of an example in proposition 2 .

Proposition 2 also shows that the positive relation between the benefits from information sharing and the number of unobserved firms can generate incomplete stable information structure, in which a group of firms agree to share information, excluding the other firms from the agreement. Proposition 3 shows, however, that such incomplete structures are never the unique stable networks, since the complete network, in which complete pooling of information occurs, is always pairwise stable.

The paper is organized as follows. Section 2 presents Raith model on information sharing, and a few new concepts and definitions due to the presence of bilateral agreements. Section 3 presents the main results, contained in propositions 1, 2 and 3; section 4 concludes.

\section{The Model}

We consider a stochastic oligopoly model with $n$ firms, in which the state of the world $\tau=\left(\tau_{1}, \tau_{2}, \ldots, \tau_{n}\right)$ represents deviations from the means of either the marginal costs or the intercept of the demand function of each firm $i$. Each $\tau_{i}$ is a random variable distributed normally, with zero mean, variance $t_{s}$ and covariance $t_{n} \geq 0$.

Each firm $i$ receives a private noisy signal $y_{i}$ about the state of the world $\tau_{i}$, with $y_{i}=$ $\tau_{i}+\eta_{i}$, where the noise $\eta_{i}$ is normally distributed with zero mean, variance $u$ and covariance $u_{n} \geq 0$. Raith (1996) defines three classes of situations with respect to information: "common value" when $t_{n}=t_{s}$, "independent values" when $t_{n}=u_{n}=0$ and "perfect signals" when $u=0$.

Firms may observe other firms' signals as a result of information sharing agreements. 
We assume that such agreements are bilateral and exclusive; this means that firm $i$ is not allowed to observe firm $j$ 's signal unless it reveals its own signal to firm $j$. We do not require transitivity in sharing agreements, in the sense that information sharing between firms $i$ and $j$ and between firms $j$ and $k$ need not imply information sharing between firms $i$ and $k$. Formally, an "information structure" is given by a non directed network $g$, in which each link $i j$ denotes a bilateral information sharing agreement between firms $i$ and $j$. We denote by $g_{i} \equiv\{j: i j \in g\} \cup\{i\}$ the set of neighbours of $i$ in $g$. The information available to firm $i$ in the information structure $g$ is therefore $I_{i}(g) \equiv\left\{y_{j}: j \in g_{i}\right\}$. We will use the notation $g+i j$ to denote the network obtained by adding to $g$ the link $i j \notin g$, and $g-i j$ to denote the network obtained by severing the link $i j \in g$ from $g$.

Following Raith (1996), we formulate firm $i$ 's profit in a general manner, without referring to a specific interpretation of $\tau$. We restrict our attention to the case of Cournot competition, in which $s_{i}$ denotes the quantity set by firm $i$ :

$$
\pi_{i}=\alpha_{i}\left(\tau_{i}\right)+\sum_{j \neq i}\left(\beta_{n}-\varepsilon s_{i}\right) s_{j}+\left(\beta+\gamma_{s} \tau_{i}-\delta s_{i}\right) s_{i}
$$

The term $\alpha_{i}\left(\tau_{i}\right)$ is a function of $\tau_{i}, \delta$ is a positive parameter and $\varepsilon \in\left(-\frac{\delta}{n-1}, \delta\right]$ expresses the degree of products differentiation. When $\gamma_{s}=1$ we have demand uncertainty, while when $\gamma_{s}=-1$ we have cost uncertainty.

With each possible information structure $g$ we associate the Bayesian Nash Equilibrium of the Cournot game in which each firm $i$ sets its quantity $s_{i}$ in order to maximize its profit as given by (1), given its available information as this is determined by $i$ 's links in $g$. Formally, a Bayesian Nash equilibrium associated with $g$ is a vector $s^{*}$ such that for each firm $i, s_{i}^{*}$ solves the following problem:

$$
s_{i}^{*}=\arg \max _{s_{i} \in R_{+}} E_{\tau, \eta}\left[\pi_{i}\left(s_{i}, s_{-i}^{*}\right) \mid I_{i}(g)\right] .
$$

The reaction function of firm $i$ as a function of $i$ 's information structure is:

$$
s_{i}=\frac{1}{2 \delta}\left(\beta+\gamma_{s} E\left[\tau_{i} \mid I_{i}(g)\right]-\varepsilon \sum_{j \neq i} E\left[s_{j} \mid I_{i}(g)\right]\right) .
$$

Standard results (see Radner, 1962 and Proposition 3.1 in Raith (1996)) can be used to establish the existence of a unique Bayesian Nash Equilibrium for all information structures 
g. Firms' Bayesian equilibrium strategies are affine in the observed signals:

$$
s_{i}^{*}=a_{i}+\sum_{j \in I_{i}(g)} b_{i j} s_{j}, \quad i=1,2, \ldots n .
$$

The $a_{i}$ and $b_{i j}$ coefficients can be computed by solving the following system, which immediately points to the main forces at work within a given information structure:

$$
\begin{aligned}
a_{i} & =\frac{1}{2 \delta}\left(\beta-\varepsilon \sum_{j \neq i} a_{j}\right) ; \\
b_{i i} & =\frac{1}{2 \delta}\left(\gamma_{s} K_{1}^{i}-\varepsilon\left(\sum_{j \in g_{i} \backslash\{i\}} b_{j i}+\sum_{z \notin g_{i}} \sum_{j \in g_{z}} K_{3}^{i} b_{j z}\right)\right) ; \\
b_{i h} & =\frac{1}{2 \delta}\left(\gamma_{s} K_{2}^{i}-\varepsilon\left(\sum_{j \in g_{h} \backslash\{i\}} b_{j h}+\sum_{z \notin g_{i}} \sum_{j \in g_{z}} K_{3}^{i} b_{j z}\right)\right) .
\end{aligned}
$$

The coefficients $K_{1}^{i}, K_{2}^{i}$ and $K_{3}^{i}$ describe the way in which firms use their observed signals to update their beliefs. In particular, $K_{1}^{i}$ and $K_{2}^{i}$ are applied to $y_{i}$ and to all $y_{j} \neq y_{i}$ such that $y_{j} \in I\left(g_{i}\right)$, respectively, to take the expectation of $\tau_{i}$, while $K_{3}^{i}$ is applied to all $y_{j} \in I\left(g_{i}\right)$ to take the expectation on the signals $y_{h}$, for all $h \notin I\left(g_{i}\right)$. Formally we have:

$$
\begin{aligned}
K_{1}^{i} & =\frac{\left(t_{s}+u+\left(\left|g_{i}\right|-2\right)\left(t_{n}+u_{n}\right)\right) t_{s}-\left(t_{n}+u_{n}\right) t_{n}\left(\left|g_{i}\right|-1\right)}{\left(t_{s}-t_{n}+u-u_{n}\right)\left(\left(t_{s}+u\right)+\left(\left|g_{i}\right|-1\right)\left(t_{n}+u_{n}\right)\right)} \\
K_{2}^{i} & =\frac{\left(t_{s}+u+\left(\left|g_{i}\right|-2\right)\left(t_{n}+u_{n}\right)\right) t_{n}-\left(t_{n}+u_{n}\right)\left(t_{s}+\left(\left|g_{i}\right|-2\right) t_{n}\right)}{\left(t_{s}-t_{n}+u-u_{n}\right)\left(\left(t_{s}+u\right)+\left(\left|g_{i}\right|-1\right)\left(t_{n}+u_{n}\right)\right)} \\
K_{3}^{i} & =\frac{\left(t_{n}+u_{n}\right)}{\left(\left|g_{i}\right|-1\right)\left(t_{n}+u_{n}\right)+t_{s}+u} .
\end{aligned}
$$

The difference in firm $i$ 's expected profit in the information structures $g$ and $g^{\prime}$ can be expressed as follows (see also Raith (1996), page 274):

$$
E \pi_{i}(g)-E \pi_{i}\left(g^{\prime}\right)=\delta\left[\operatorname{var}\left(s_{i}^{*}(g)-\operatorname{var}\left(s_{i}^{*}\left(g^{\prime}\right)\right)\right]\right.
$$

where in (10) we have used the fact that the terms $a_{i}$ in the equilibrium strategies are the same for all firms $i$ and for all information structures.

\section{Information Sharing in Common Value Games}

We study the incentives to share information in common value situations $\left(t_{s}=t_{n}\right)$ when sharing agreements are bilateral and exclusive. The main results in the literature for the 
case of exclusive agreements refers to the specific contract in which firms choose whether to universally share information (the complete network in the present setting) or not to share any (the empty network in the present setting). These results are well summarized by Proposition 4.4 in Raith (1996), rephrased below in the terminology of this paper:

In a Common Value information sharing situation, the expected profits of firms in the complete network is larger than the expected profits in the empty network if $\mu \equiv \frac{\varepsilon}{\delta}<\frac{2}{n+1}$, is smaller if $\mu>\frac{2}{\sqrt{n}+1}$, and may be either larger or smaller in the range $\frac{2}{n+1}<\mu<\frac{2}{\sqrt{n}+1}$, depending on the parameters of the model.

Note that $\varepsilon$ is an index of products' differentiation, and that when $\varepsilon<0$ strategies are complements, while $\varepsilon>0$ is the case of strategic substitutes. So, complete pooling of information is never profitable when strategies are substitutes and products are homogeneous, while it is always profitable when products are extremely differentiated and/or strategies are complements.

In the present context of bilateral information sharing, we are not only interested in comparing the complete and the empty networks, and we allow for any incomplete structure where information sharing is partial. In order to identify equilibrium information structures, we borrow a well known stability notion from the theory of strategic network formation. We say that the network $g$ is a pairwise stable information structure if it satisfies two conditions: no firm has an incentive to sever one of its links in $g$ and no pair of firms have an incentive to add a new link to $g$.

Definition 1 The information structure $g$ is pairwise stable if:

1) $E \pi_{i}(g) \geq E \pi_{i}(g-i j)$ for all $i j \in g$;

2) $E \pi_{i}(g+i j)>E \pi_{i}(g) \rightarrow E \pi_{j}(g+i j)<E \pi_{j}(g)$ for all $i j \notin g$.

Note that the empty network is not pairwise stable if there exists a pair of firms that would be better off by mutually exchanging their private information. The next proposition shows that such incentives may exist even when strategies are substitutes and products are homogeneous, and always exist when the number of firms in large enough (the proof of all results in the paper are found in the appendix).

Proposition 1 Consider a common value information sharing situation.

i) If $\mu<\frac{2}{3}$ the empty network is never pairwise stable, for all values of $n$. 
ii) If $\frac{2}{3}<\mu<\frac{2}{1+\sqrt{2}}$ then there exists a $p_{n}^{* *}$ such that for all $p_{n}<p_{n}^{* *}$ the empty network is never pairwise stable, for all values of $n$; otherwise (when $p_{n}>p_{n}^{* *}$ ) there exists a finite number of firms $n^{*}$ such that for all $n>n^{*}$ the empty network is not pairwise stable.

iii) If $\mu>\frac{2}{1+\sqrt{2}}$ there exists $p_{n}^{*}$ and a finite value $n^{*}$ such that for all $p_{n}>p_{n}^{*}$ and $n>n^{*}$ the empty network is not pairwise stable.

It is useful to discuss the forces behind propositions 1 and 2 comparing our results with Raith's (1996) results for the contractual model. Point i) covers those cases in which two firms always have an incentive to share information when no other firm is in the market $\left(\frac{2}{3}\right.$ is in fact $\frac{2}{n+1}$ for $\left.n=2\right)$. Point $i$ ) shows that these incentives remain when there are more than two firms in the market. Points ii) and iii) cover situations in which two firms may not have the incentive to share information when alone in the market. Point ii) shows that when these incentives exist (low $p_{n}$ ), they do not vanish as we add firms to the market. More interestingly, when such incentives are absent when no other firms are in the market (high $p_{n}$ ), these incentives are shown to appear as we add more firms in the market. Finally, point iii) refers to those problems in which two firms would never share information, for any value of $p_{n}$ (these problems include the standard Cournot game with homogeneous products). Here, it is shown that by adding firms in the market we can generate incentives for bilateral information sharing, provided the correlation parameter $p_{n}$ is large enough.

Note that the threshold levels of $\mu$ is Raith's paper are decreasing in $n$. Therefore, it is not possible that by adding firms in the market we pass from a situation where the empty graph dominates the complete graph to a situation where the opposite is true. In other words, the results of points ii) and iii) show that incentives for bilateral information sharing exist in problems where complete information pooling is not profitable, and are responsible for the instability of the empty network.

The mechanics underlying these results have been briefly discussed in the introduction. When two firms share information in a market where other firms exist, they not only obtain a refined information on the state of the world and the observation of the other firm's signal, but also obtain a refined information on the signals observed by the other firms in the market, through signals' correlation. The larger the number of these firms, the larger the benefits for the two sharing firms in terms of expected profits. Not surprisingly, at point iii), where the dominance of the empty network on the complete network is more severe for $n=2$, our result requires a sufficiently large correlation of firms signals. 
Note finally how the number of firms in the market play opposite roles in the present paper and in the previous literature. While in Raith's paper a larger $n$ enlarges the set of problems in which the absence of information is profitable with respect to an industry-wide information sharing agreement, in the present paper a larger $n$ increases the benefits from bilateral information sharing, making the empty network unstable.

Proposition 2 below provides an example in which information is shared in equilibrium even with the minimal number of firms that is required to generate the beneficial effect underlying the result of proposition 1. Proposition 2 also shows that equilibrium information structures need not entail either the absence or the complete pooling of information, but may disclose part of the information in the system to a subset of firms.

Proposition 2 There exist common value problems that admit an incomplete stable information structure.

It is useful to sketch here the example used to prove Proposition 2 in the appendix. Firms 1 and 2 find it profitable to share information (an example of proposition 1 at work in which the required level of $n$ is $n^{*}=2$ ), but neither have the incentive to form a link with 3 , on which they already acquired enough information through the link 12 . The result is obtained for a high level of signals' errors' correlation $u_{n}$, ensuring at the same time the incentives to form the link 12 and not to form a link with firm 3. Firm 3 is actually excluded from information sharing, since it would have an incentive to form a link with either 2 or 1 .

Proposition 2 also implies that the complete network may not always be achieved though a dynamic process of formation of bilateral agreements. However, in the example of proposition 2 the complete network is pairwise stable, since no firm has an incentive to sever one of its links. The next final proposition shows that this is a very general feature of all common value situations.

Proposition 3 Let $n \geq 3$. The complete network is always a pairwise stable information structure.

We finally remark that the stability result of proposition 3, at least in its general form, is very specific to the concept of pairwise stability, allowing only for one-link deviations. A more sever requirement for stability would allow firms to sever all their links at a time, withdrawing all their private information from all competitors. Although it can be checked 
that the complete network satisfies this stronger requirement in the example studied by proposition 2, other instances of Cournot competition can be constructed in which it is not so. In contrast, the results of both proposition 1 and 2 would carry over to this stronger notion of stability.

\section{Conclusions}

We have studied information sharing in oligopoly when firms can make bilateral and exclusive agreements. We have shown that some amount of information is always shared in equilibrium, even in common value situations with strategic substitutes, for which the previous literature, allowing only for an industry-wide agreement, has predicted the complete absence of information sharing. We have also shown that it is possible to construct symmetric situations in which some but not all information is shared in equilibrium, and some firms are excluded from the group of information sharing firms. The implications for policy are quite relevant and partly contrast with those inspired by previous works on the subject, since evidence of information sharing need not be associated with collusive behaviour.

\section{References}

[1] Clarke, R., Collusion and the incentives for information sharing, Bell J. Econ. 14 (1983), 383-394.

[2] Gal-Or, E., Information sharing in oligopoly, Econometrica 53 (1985), 329-343.

[3] Gal-Or, E., Information transmission - Cournot and Bertrand equilibria, Rev. Econ. Stud. 53 (1986), 85-92.

[4] Kirby, A. J., Trade associations information exchange mechanisms, RAND J. Econ. 19 (1988), 138-146.

[5] Kühn, K. U., and X. Vives, Information Exchanges among Firms and their Impact on Competition, Office for Official Publications for the European Community, Luxemburg, 1995. 
[6] Jackson, M. O., and A. Wolinsky, A strategic model of social and economic networks, $J$. Econ. Theory $\mathbf{7 1}$ (1996), 44-74.

[7] Novshek, W. and H. Sonnenschein, Fulfilled expectations Cournot duopoly with information acquisition and release, Bell J. Econ. 13 (1982), 214-218.

[8] Radner, R., Team decision problems, Annals Math. Statist. 33 (1962), 857-81.

[9] Raith, M., A general model of information sharing in oligopoly, J. Econ. Theory 71 (1996), 260-288.

[10] Vives, X., Duopoly information equilibrium: Cournot and Bertrand, J. Econ. Theory 34 (1984), 71-94.

[11] Vives, X., Trade association disclosures rules, incentives to share information and welfare, Rand J.Econ. 21 (1990), 409-430.

\section{APPENDIX}

\section{Proof of proposition 1.}

The proof is organized in several steps, and goes by studying the difference in expected profits of two firms, 1 and 2, in the empty networks $g^{\varnothing}$ and in the network $g^{12} \equiv\{12\}$. Denoting by $a$ and $b$ the coefficients of equilibrium strategies in the empty network and by $a_{1}=a_{2}, b_{11}=b_{12}=b_{21}=b_{22}$ the equilibrium coefficients of firms 1 and 2 in the network $g^{12}$, we obtain:

$$
\begin{aligned}
a_{1} & =a_{2}=a=\frac{\beta}{2 \delta+(n-1) \varepsilon} \\
b & =\frac{\left(K_{1}^{1}\right)^{2}\left(t_{s}+u\right) \gamma_{s}^{2}}{2 \delta+K_{3}^{1} \varepsilon(n-1)} \\
b_{11} & =b_{12}=b_{21}=b_{22}=\frac{\left.\gamma_{s}\left(2 K_{1}^{2} \delta+K_{1}^{2} K_{3}^{1}(n-3) \varepsilon\right)-K_{1}^{1} K_{3}^{2}(n-2) \varepsilon\right)}{4 \delta^{2}+2\left(1+K_{3}^{1}(n-3)\right) \delta \varepsilon+K_{3}^{1}\left(n-3-4 K_{3}^{2}(n-2)\right) \varepsilon^{2}} .
\end{aligned}
$$

From (10), we can express the difference in profits of firm 1 (and, by symmetry, of firm 2) in $g^{\varnothing}$ and in $g^{12}$ as the difference of the variances of equilibrium quantities:

$$
E \pi\left(g^{\varnothing}\right)-E \pi\left(g^{12}\right)=b^{2}\left(t_{s}+u\right)-2 b_{11}^{2}\left(2 t_{s}+u+u_{n}\right) .
$$


Plugging in (11) the values of the $K$ 's and the $b$ 's coefficients, denoting by $p_{i}=\left(t_{s}+u\right)$ and by $p_{n}=\left(t_{s}+u_{n}\right)$ the variance and covariance of signals, and letting $p \equiv p_{i}+p_{n}$, we obtain the following expression:

$$
\begin{aligned}
E \pi\left(g^{\varnothing}\right)-E \pi\left(g^{12}\right)= & t^{2} \gamma_{s}^{2}\left[\frac{p_{i}}{\left(2 p_{i} \delta+(n-1) p_{n} \varepsilon\right)^{2}}\right. \\
& \left.-\frac{2 p\left(p_{n} \varepsilon-2 p_{i} \delta\right)^{2}}{\left(4 p_{i} p \delta^{2}+2 p\left(p_{i}+(n-3) p_{n}\right) \delta \varepsilon-p_{n}\left((3 n-5) p_{n}-(n-3) p_{i}\right) \varepsilon^{2}\right)^{2}}\right]
\end{aligned}
$$

It can be shown that the denominator of (12) is always strictly positive for all admissible values of the parameters (a complete proof of this fact is available on request). The sign of (11) is therefore the same as the sign of the numerator of (12). Dividing the numerator of (12) by $\delta^{4}$ and denoting $\frac{\varepsilon}{\delta}$ by $\mu$ we obtain the following expression:

$$
a \cdot n^{2}+b \cdot n+c
$$

where

$$
\begin{aligned}
a= & \left(p_{i}-p_{n}\right) p_{n}^{2} \mu^{2}\left(4 p_{i} p(\mu-1)+\left(p_{i}^{2}-5 p_{i} p_{n}+2 p_{n}^{2}\right) \mu^{2}\right) \\
b= & 2\left(p_{i}-p_{n}\right) p_{n} \mu\left(-8 p_{i}^{2} p+4 p_{i} p\left(2 p_{i}+3 p_{n}\right) \mu+2 p_{i}\left(p_{i}-8 p_{n}\right) p \mu^{2}-p_{n}\left(3 p_{i}^{2}-11 p_{i} p_{n}+2 p_{n}^{2}\right) \mu^{3}\right) \\
c= & \left(p_{i}-p_{n}\right)\left[2 p_{n}^{4} \mu^{4}+p_{i} p_{n}^{3} \mu^{2}((44-21 \mu) \mu-36)+4 p^{4}(\mu(4+\mu)-4)\right. \\
& \left.-4 p_{i}^{3} p_{n}(\mu-1)(3 \mu(4+\mu)-4)+p_{i}^{2} p_{n}^{2} \mu(48+\mu(\mu(32+9 \mu)-76))\right]
\end{aligned}
$$

We first record the following two facts:

E1) setting $n=2$ we obtain ${ }^{3}$ that the sign of (12) is negative for $\mu<\frac{2}{3}$, is positive for $\mu>\frac{2}{1+\sqrt{2}}$ and in the intermediate range is positive if and only if $p_{n}>\frac{p_{i}\left(4-4 \mu-\mu^{2}\right)}{2 \mu^{2}} \equiv p_{n}^{* *}$.

E2) the roots $\left(n_{-}, n_{+}\right)$of $(12)$ are real (since $\left.b^{2}-4 a c \geq 0\right)$, distinct and finite as long as $a \neq 0$.

To continue the proof we need of the following lemmas:

Lemma 1 1) if $\mu<\frac{2}{1+\sqrt{2}}$ then (12) is concave. 2) if $\mu>\frac{2}{1+\sqrt{2}}$ then there exists $p_{n}^{*}$ such that for all $p_{n}>p_{n}^{*}$ (12) is concave, otherwise it is convex.

\footnotetext{
${ }^{3}$ These are same stability conditions as in Raith (1996), proposition 4.4: in fact, for $n=2$, the formation of the link 12 is equivalent to complete pooling of information.
} 
Proof of Lemma 1: Note that concavity of (12) depends on the sign of term $a$, that is the same of the term:

$$
\left(4 p_{i} p(\mu-1)+\left(p_{i}^{2}-5 p_{i} p_{n}+2 p_{n}^{2}\right) \mu^{2}\right)
$$

Let us evaluate the roots of this last term as a function of $p_{n}$. We find:

$$
\frac{4 p_{i}(1-\mu)+5 p_{i} \mu^{2} \pm p_{i}(\mu-2) \sqrt{4(1-\mu)+17 \mu^{2}}}{4 \mu^{2}}
$$

The largest root yields a value which exceeds $p_{i}$. We therefore consider only the smaller root $p_{n}^{*}$, and conclude that if (13) is a convex function of $p_{n}$, then $a$ is negative for all $p_{n}>p_{n}^{*}$. Since it can be easily checked that the second derivative of $a$ with respect to $p_{n}$ has the same sign as $\mu^{2}$, we conclude that $a$ is negative for values of $p_{n}$ larger than the smaller root $p_{n}^{*}$. Now we show that if $\mu<\frac{2}{1+\sqrt{2}}$ then $p_{n}^{*}<0$, implying, by virtue of step above, that $a<0$ for all parameters' values. Otherwise, when $\mu>\frac{2}{1+\sqrt{2}}, p_{n}^{*}>0$ and increasing in $\mu$. In this case, $a<0$ for all values $p_{n}^{*}<p_{n}<p_{i}$. Consider again the smaller root in (14)

$$
p_{n}^{*}=\frac{4 p_{i}(1-\mu)+5 p_{i} \mu^{2}+p_{i}(\mu-2) \sqrt{4(1-\mu)+17 \mu^{2}}}{4 \mu^{2}} .
$$

Solving 15 with respect to $\mu$ we obtain that it is equal to zero for:

$$
\mu_{-}=2(-1-\sqrt{2}) ; \quad \mu_{+}=\frac{2}{1+\sqrt{2}} .
$$

Computing the first derivative of (15) with respect to $\mu$ we obtain a strictly positive value for all $\mu$ in the range $(0,1]$. This implies that for all $0<\mu<\mu_{+}$we have $p_{n}^{*}<0$. Also, we have that $p_{i}>p_{n}^{*}>0$ for $1 \geq \mu>\mu_{+}$. Finally we note that $\mu_{-}$is out of the allowed range $\left(-\frac{1}{n-1}, 1\right]$ of $\mu$; moreover computing the first derivative of (15) with respect to $\mu$ we obtain a strictly negative value for all $\mu$ in the range $\left(\mu_{-}, 0\right)$. Then for all $-\frac{1}{n-1}<\mu<\mu_{+}$we have $p_{n}^{*}<0$, that means $a<0$. QED

Lemma 2 Let $n=2$. 1) If $\mu>0$, there exists $\hat{p}_{n}>0$ such that (12) is increasing in $n$ if $p_{n}<\hat{p}_{n}$ and $\mu>\frac{2}{1+\sqrt{2}}$, otherwise (12) is decreasing in n; 2) If $\mu<0$ then (12) is increasing in $n$.

Proof of Lemma 2: The first derivative of (12) at $n=2$ is given by:

$$
2\left(p_{i}-p_{n}\right) p_{n} p \mu\left(2 p_{i}-p_{n} \mu\right)\left(p_{i}(\mu(4+\mu)-4)-2 p_{n} \mu^{2}\right)
$$


The sign of (17) is the same as the sign of the following expression:

$$
\mu\left(p_{i}(\mu(4+\mu)-4)-2 p_{n} \mu^{2}\right)
$$

The expression in brackets in (18) is positive for $p_{n}<\frac{p_{i}(\mu(4+\mu)-4)}{2 \mu^{2}}$. It is directly verifiable that the right side of this inequality will take negative values for $\mu<\frac{2}{1+\sqrt{2}}$ and positive values for $\mu>\frac{2}{1+\sqrt{2}}$. The results follows. QED.

We are now ready to prove points $i$ )-iii) of the proposition.

Point i) $\left(\mu<\frac{2}{3}\right)$. The proof of this part is organized in two steps.

Step 1. $(\mu<0)$. We know that at $n=2(12)$ is negative (by E1) and increasing (by Lemma 2); moreover we know that (12) is concave (by Lemma 1). These facts tell us that the two real roots of $(12)\left(n_{-}, n_{+}\right)$are larger than 2 . Now we show that $\left(n_{-}, n_{+}\right)$are larger than the admissible value of $n$ for any $\mu<0$.

It is directly verifiable that when $\mu<0, n<1-\frac{1}{\mu}$.

We find that the difference between the smaller root $n_{-}$and the maximum allowed value of $n$ is positive for all $\mu<0$. It follows that (12) remains negative for all admissible values of $n$, which means that the empty network is not pairwise stable.

Step 2. $\left(0<\mu<\frac{2}{3}\right)$. We know that at $n=2$ (12) is decreasing (Lemma 2); moreover we know that (12) is concave (Lemma 1). This two facts tell us the all points $n \geq 2$ are in the right (and decreasing) branch of the (parabola) (12). By E1 we know that (12) takes negative values at $n=2^{4}$; Therefore these evidences are enough to show that (12) remains negative for all $n \geq 2$.

Point ii) $\left(\frac{2}{3}<\mu<\frac{2}{1+\sqrt{2}}\right)$. We know that at $n=2$ (12) is decreasing (Lemma 2); moreover we know that (12) is concave (Lemma 1). These two facts tell us the all points $n \geq 2$ are in the right (and decreasing) branch of the parabola given by (12). Now suppose that (12) takes negative values at $n=2 ;^{5}$ the two real roots of (12) are strictly smaller than 2 ; it follows that (12) remains negative for all $n \geq 2$. Now suppose that (12) takes positive values at $n=2 ;{ }^{6}$ the larger real root $n_{+}$must be larger than 2 ; it follows that, for all $n>n_{+}$ (12) takes negative values.

\footnotetext{
${ }^{4}$ by E1, it happens when either $\mu>\frac{2}{1+\sqrt{2}}$ or $\frac{2}{3}<\mu<\frac{2}{1+\sqrt{2}}$ and $p_{n}>\frac{p_{i}\left(4-4 \mu-\mu^{2}\right)}{2 \mu^{2}}$.

${ }^{5} \mathrm{By} \mathrm{E} 1$, it happens when either $\mu<\frac{2}{3}$ or $\frac{2}{3}<\mu<\frac{2}{1+\sqrt{2}}$ and $p_{n}<\frac{p_{i}\left(4-4 \mu-\mu^{2}\right)}{2 \mu^{2}}$.

${ }^{6} \mathrm{By}$ E1, it happens when either $\mu>\frac{2}{1+\sqrt{2}}$ or $\frac{2}{3}<\mu<\frac{2}{1+\sqrt{2}}$ and $p_{n}>\frac{p_{i}\left(4-4 \mu-\mu^{2}\right)}{2 \mu^{2}}$.
} 
Point iii) $\left(\mu>\frac{2}{1+\sqrt{2}}\right)$. By lemma 1 we know that (12) is concave if $p_{n}>p_{n}^{*}$, and otherwise is convex; moreover we know that at $n=2(12)$ is positive (by E1). Now suppose (12) is concave (that is, $p_{n}>p_{n}^{*}$ ): the larger real root $n_{+}$must be larger than 2 ; it follows that, for all $n>n_{+}$(12) takes negative values. Now suppose (12) is convex (that is $p_{n}<p_{n}^{*}$ ); by lemma 2 we know that at $n=2$ the function (12) is increasing in $n$ if $p_{n}<\hat{p}_{n}$ (note that we find that $\hat{p}_{n}=p_{n}^{*}$ when $\mu=\frac{2}{1+\sqrt{2}}$; moreover, we find that for $\mu>\frac{2}{1+\sqrt{2}}, \hat{p}_{n}-p_{n}^{*}$ is increasing in $\mu^{7}$; it follows that $p_{n}^{*}<\hat{p}_{n}$ so that we have $\left.p_{n}<p_{n}^{*}<\hat{p}_{n}\right)$. Therefore the two real roots $\left(n_{-}, n_{+}\right)$must be smaller than 2 and the (12) takes positive values for $n \geq 2$.

Proof of proposition 2. We develop a simple Cournot example in which three firms are endowed with symmetric private information (same $u_{n}$ and $u_{i}$ ) and produce slightly differentiated products $(\varepsilon=0.9)$ in a common market with demand uncertainty $\left(\gamma_{s}=1\right)$. For simplicity we neglect costs, and we let $\alpha_{i}\left(\tau_{i}\right)=0$ for all $i, \beta_{n}=0$ and $\delta=1$. We also set $t_{s}=u=1$. We show that the network $g^{*}=\{12\}$ is pairwise stable.

We first compute equilibrium strategies and expected profits in $g^{*}$. The updating coefficients in $g^{*}$ are (see expressions (7)-(9)):

$$
\begin{aligned}
& K_{1}^{1}=K_{2}^{1}=K_{1}^{2}=K_{2}^{2}=K_{1}^{3}=K_{2}^{3}=\frac{1}{\left(2+\left(1+u_{n}\right)\right)} \\
& K_{3}^{1}=K_{3}^{2}=K_{3}^{3}=\frac{\left(1+u_{n}\right)}{3+u_{n}} .
\end{aligned}
$$

The coefficient of firms' equilibrium strategies in the Bayesian game associated with $g^{*}$ are (see expressions (5)-(6):

$$
\begin{aligned}
& a_{11}=a_{12}=\frac{\beta}{2+2 \varepsilon} \\
& b_{11}=b_{21}=b_{12}=b_{22}=\frac{2 K_{1}^{1}-K_{1}^{1} K_{3}^{2} \varepsilon}{2+\varepsilon-2\left(K_{3}^{1} \varepsilon\right)^{2}} \\
& b_{33}=\frac{4 K_{1}^{2} K_{3}^{1} \varepsilon-K_{1}^{1}(2+\varepsilon)}{4\left(K_{3}^{1} \varepsilon\right)^{2}-2(2+\varepsilon)}
\end{aligned}
$$

In order to evaluate the stability of the information structure $g^{*}$, we need to evaluate the equilibria and the expected profits associated with the two networks $g^{\varnothing}$ (that originates by

\footnotetext{
${ }^{7}$ More precisely, the first derivative of the expression $\frac{p_{i}(\mu(4+\mu)-4)}{2 \mu^{2}}-p_{n}$ is increasing in $\mu$.
} 
severing the link 12 from $g^{*}$ ) and the network $g^{\prime}=\{12,23\}$ (that originates by adding the link 23 to $\left.g^{*}\right)$. For $g^{\varnothing}$ we obtain:

$$
\begin{aligned}
& K_{1}^{1}=K_{1}^{2}=K_{1}^{3}=\frac{\left(1+u_{n}\right)}{\left(1-u_{n}\right)^{2}} ; \\
& K_{3}^{1}=K_{3}^{2}=K_{3}^{3}=\frac{\left(1+u_{n}\right)}{2} .
\end{aligned}
$$

and:

$$
\begin{aligned}
a & =\frac{\beta}{2+2 \varepsilon} \\
b & =\frac{K_{1}^{1}}{2\left(1+K_{3}^{1} \varepsilon\right)} .
\end{aligned}
$$

For $g^{\prime}$ we obtain::

$$
\begin{gathered}
K_{1}^{1}=K_{2}^{1}=K_{1}^{3}=K_{2}^{3}=\frac{1}{\left(2+\left(1+u_{n}\right)\right)} \\
K_{1}^{2}=K_{2}^{2}=\frac{2-\left(1+u_{n}\right)}{\left(1-u_{n}\right)\left(4+2 u_{n}\right)} \\
K_{3}^{1}=K_{3}^{3}=\frac{\left(1+u_{n}\right)}{3+u_{n}} \\
K_{3}^{2}=\frac{\left(1+u_{n}\right)}{2\left(2+u_{n}\right)}
\end{gathered}
$$

and:

$$
\begin{aligned}
& b_{11}=b_{33}=\frac{2 K_{1}^{2}-\varepsilon K_{1}^{3}-K_{1}^{3} K_{3}^{2} \varepsilon}{(2-\varepsilon)\left(2+\varepsilon+K_{3}^{2} \varepsilon\right)} \\
& b_{12}=b_{32}=\frac{2 K_{2}^{2}\left(2+\varepsilon-2 K_{3}^{2}+2 \varepsilon K_{3}^{2}\right)-K_{1}^{3}\left(2+2 \varepsilon K_{3}^{2}+K_{1}^{3} \varepsilon^{2}+K_{3}^{2} \varepsilon^{2}\right)}{2(2-\varepsilon)(1+\varepsilon)\left(2+\varepsilon+\varepsilon K_{3}^{2}\right)} \\
& b_{21}=b_{23}=\frac{K_{1}^{3}\left(2+\varepsilon K_{3}^{2}\right)-\varepsilon K_{1}^{2}}{(2-\varepsilon)\left(2+\varepsilon+\varepsilon K_{3}^{2}\right)} \\
& b_{22}=\frac{K_{1}^{3}\left(4+4 \varepsilon+2 \varepsilon K_{3}^{2}+\varepsilon^{2}+3 K_{3}^{2} \varepsilon^{2}\right)-K_{2}^{2}\left(4 \varepsilon+2 \varepsilon^{2}\right)}{2\left(2+\varepsilon+\varepsilon K_{3}^{2}\right)\left(2-\varepsilon+\varepsilon^{2}\right)} \\
& a_{1}=a_{2}=a_{3}=\frac{\beta}{2+2 \varepsilon}
\end{aligned}
$$

From (10), in order to compare firms' expected profits in these three structures we only need to look at the variance of their equilibrium strategies (note, in fact, that the term $a$ the intercept of the equilibrium strategy - is the same in all three networks and for all firms). We obtain the following expressions: 


$$
\begin{aligned}
E \pi_{1}\left(g^{*}\right) & =E \pi_{2}\left(g^{*}\right)=2\left(2 b_{11}\right)^{2}+2 b_{11}^{2}\left(1+u_{n}\right) \\
E \pi_{3}\left(g^{*}\right) & =2 b_{33}^{2} \\
E \pi_{i}\left(g^{\varnothing}\right) & =2 b^{2}, i=1,2,3 \\
E \pi_{2}\left(g^{\prime}\right) & =2\left(2 b_{21}^{2}+b_{21}^{2}\right)+2 b_{21} b_{21}\left(1+u_{n}\right)+2 b_{23} b_{22}\left(1+u_{n}\right)+2 b_{21} b_{23}\left(1+u_{n}\right) \\
E \pi_{3}\left(g^{\prime}\right) & =2\left(b_{11}^{2}+b_{12}^{2}\right)+2 b_{11}^{2}\left(1+u_{n}\right)
\end{aligned}
$$

We show that for high enough values of $u_{n}$ (the correlation of the error term of firms' signals), the network $g^{*}$ satisfies the following conditions and is therefore pairwise stable:

$$
\begin{aligned}
& E \pi_{1}\left(g^{*}\right)>E \pi_{i}\left(g^{\varnothing}\right) \\
& E \pi_{2}\left(g^{*}\right)>E \pi_{2}\left(g^{\prime}\right) \\
& E \pi_{3}\left(g^{\prime}\right)>E \pi_{3}\left(g^{*}\right) .
\end{aligned}
$$

Although the expressions of profits are quite long and complex, the result can be easily illustrated by means of three pictures.

Figure 1 reports the difference $E \pi_{i}\left(g^{\varnothing}\right)-E \pi_{1}\left(g^{*}\right)$

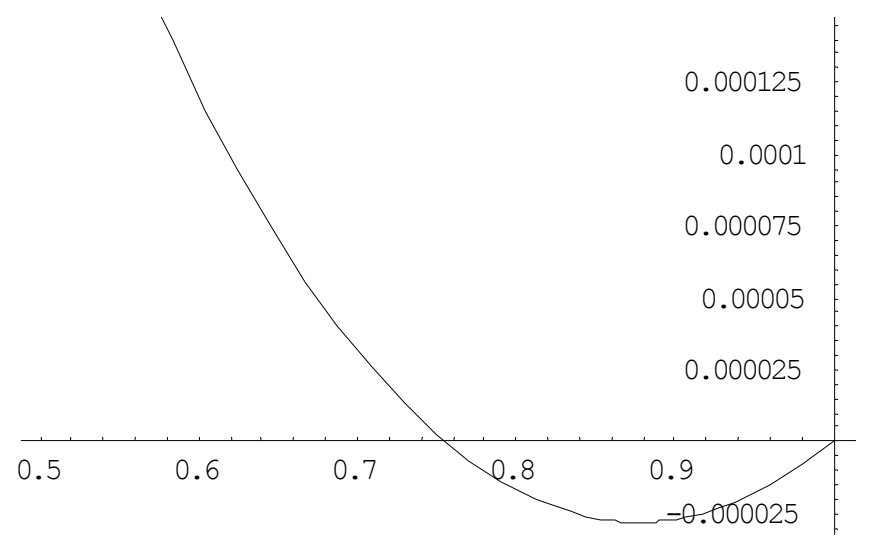

Figure 1. Incentives to sever link 12.

This difference is negative for high enough values of $u_{n}$, meaning that for high enough correlation of signals, firms 1 and 2 have no incentives to sever their link in $g^{\prime}$. This result is a specific case of proposition 2 , in which the value of $n$ which is sufficient to create incentives to form a link starting from the empty network is $n=3$. 
Figure 2 reports the difference $E \pi_{2}\left(g^{\prime}\right)-E \pi_{2}\left(g^{*}\right)$ as a function of $u_{n}$.

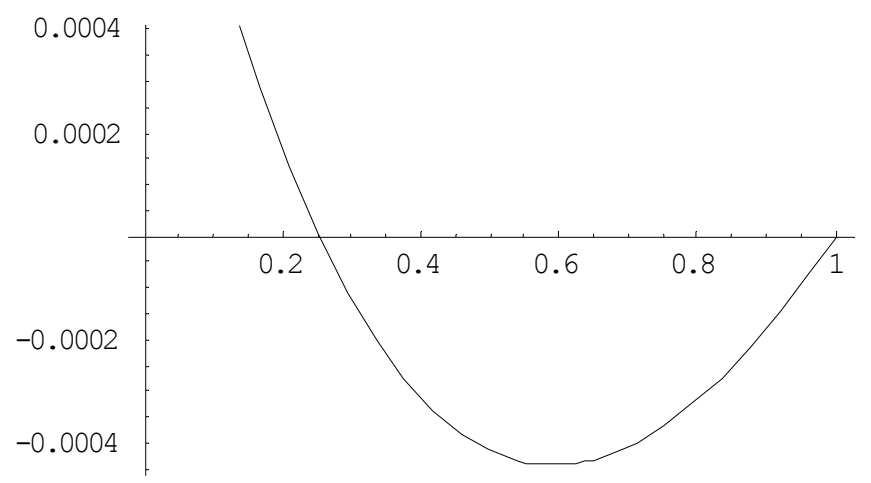

Figure 2. Incentive of firm 2 to form the link 23.

For high enough values of signals' correlation, firm 2 has no incentives to form the link 23 starting from $g^{*}$. This result has an intuitive interpretation: when correlation among signals is not too low, firms 1 and 2 are able to infer enough information on firm 3's signal by observing each other's signals. It follows that neither firm is willing to disclose to agent 3 its own private information (that is, to form a link with 3 ) in order to obtain additional information on firms 3's signal. Note that firms 3 is actually excluded from the information sharing group made of firms 1 and 2. In fact, it can be shown that condition (43) holds, that is, that firm 3 would indeed be willing to link and form the link 23. This is illustrated in Figure 3, reporting the difference $E \pi_{3}\left(g^{*}\right)-E \pi_{3}\left(g^{\prime}\right)$, which is positive for high enough values of $u_{n}$. 


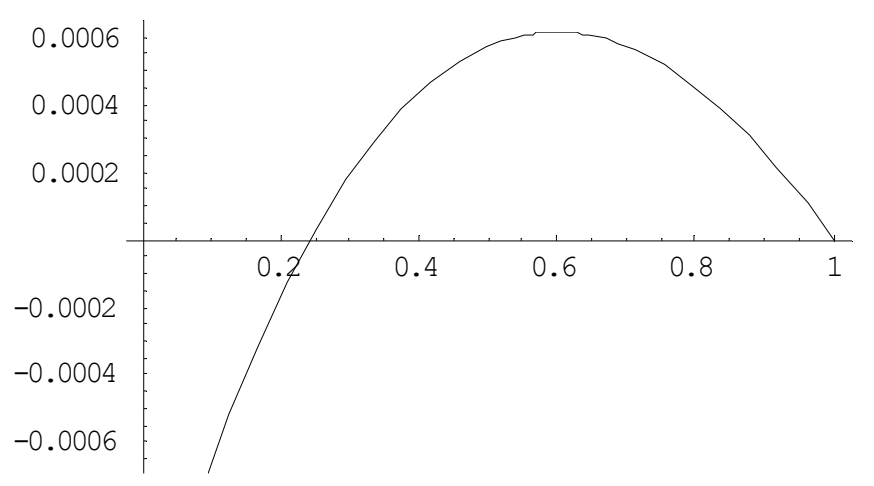

Figure 3. Incentive of firm 3 to form the link 23.

This observations conclude the proof.

\section{Proof of proposition 3}

The proof is organized in several steps, and goes by studying the difference in expected profits of two firms, 1 and 2, in the complete networks $g^{c}$ and in the network $g^{-12} \equiv\left\{g^{c}-12\right\}$. Denoting by $a$ and $b_{1}=b_{i j}$ for all $i, j \in N$ the coefficients of equilibrium strategies in the complete network and by $a_{1}=a_{2}, b_{11}=b_{22}$, and $b_{13}=b_{1 j}=b_{23}=b_{2 j}$ for all $j \in N / 1,2$ the equilibrium coefficients of firms 1 and 2 in the network $g^{-12}$, we obtain:

$$
\begin{aligned}
a_{1} & =a_{2}=a=\frac{\beta}{2 \delta+(n-1) \varepsilon} \\
b_{1} & =\frac{\gamma_{s} K_{1}^{i}}{2 \delta+(n-1) \varepsilon} \\
b_{11} & =\gamma_{s} \frac{K_{1}^{1}(2 \delta+(n-3) \varepsilon)-\left(1+K_{3}^{1}\right) K_{1}^{3}(n-2) \varepsilon}{(2 \delta-\varepsilon)\left(2 \delta+\left(n-2+K_{3}^{1}\right) \varepsilon\right)} \\
b_{13} & =\gamma_{s} \frac{(2 \delta+(n-2) \varepsilon) K_{1}^{1}(2 \delta+(n-3) \varepsilon)-\left(1+K_{3}^{1}\right) K_{1}^{3}(n-2) \varepsilon}{(2 \delta-\varepsilon)(2 \delta+(n-1) \varepsilon)\left(2 \delta+\left(n-2+K_{3}^{1}\right) \varepsilon\right)}
\end{aligned}
$$

From (10), we can express the difference $E \pi\left(g^{c}\right)-E \pi\left(g^{-12}\right)$ in the profits of firm 1 (and, by symmetry, of firm 2) in $g^{c}$ and in $g^{-12}$ as the difference of the variances of equilibrium quantities, here given by the following expression:

$$
\begin{aligned}
& n \cdot b_{1}^{2}\left(\left(t_{s}+u\right)+(n-1)\left(t_{s}+u_{n}\right)\right)-\left(b_{11}^{2}+(n-2) b_{13}^{2}\right)\left(t_{s}+u\right) \\
& -(n-2) b_{13}\left(2 b_{11}+(n-3) b_{13}\right)\left(t_{s}+u_{n}\right) .
\end{aligned}
$$


Plugging in (44) the values of the $K$ 's and the $b$ 's coefficients, denoting by $p_{i}=\left(t_{s}+u\right)$ and by $p_{n}=\left(t_{s}+u_{n}\right)$ the variance and covariance of signals, and letting $p \equiv p_{i}+p_{n}$, we obtain the following expression for the difference $E \pi\left(g^{c}\right)-E \pi\left(g^{-12}\right)$ :

$$
\frac{(p i-p n) t^{2} \gamma_{s}^{2} \delta\left(4\left(p_{i}+(n-2) p_{n}\right)\left(\delta^{2}+(n-3) \delta \varepsilon\right)+\left((7+(n-6) n) p_{i}+(n(19+(n-8) n)-16) p_{n}\right) \varepsilon^{2}\right)}{\left(p_{i}+(n-1) p_{n}\right)(2 \delta+(n-1) \varepsilon)^{2}\left(2 p_{i} \delta+2(n-2) p_{n} \delta+(n-2) p_{i} \varepsilon+(5+(n-4) n) p_{n} \varepsilon\right)^{2}} .
$$

It can be shown that the denominator of the above equation is always strictly positive for all admissible values of the parameters; moreover the sign is the same as the sign of the following expression.

$$
\left(4\left(p_{i}+(n-2) p_{n}\right)\left(\delta^{2}+(n-3) \delta \varepsilon\right)+\left((7+(n-6) n) p_{i}+(n(19+(n-8) n)-16) p_{n}\right) \varepsilon^{2}\right)
$$

We divide (46) in two terms. The first, $4\left(p_{i}+(n-2) p_{n}\right)\left(\delta^{2}+(n-3) \delta \varepsilon\right)$, is always: indeed by assumption $\varepsilon>-\frac{\delta}{n-1}$ and the proof follows directly; it can be directly verified that the second term of (46) is positive for $n \geq 5$. Therefore (46) could be negative only for $n=3$ and $n=4$. But for $n=3(46)$ becomes $4\left(p_{i}+p_{n}\right) \delta^{2}-2\left(p_{i}+2 p_{n}\right) \varepsilon^{2}$ and for $n=4$ (46) becomes $4\left(p_{i}+2 p_{n}\right)\left(\delta^{2}+\delta \varepsilon\right)-\left(p_{i}+4 p_{n}\right) \varepsilon^{2}$ and, by the assumption that $\varepsilon>-\frac{\delta}{n-1}$, both terms are strictly positive. 